\title{
The use of point of care ultrasonography in the perioperative period: A case of Takotsubo cardiomyopathy
}

\author{
Nicholas Ryan David Humphreys MD', William Neil Maxwell Gallacher MBChB \\ $\operatorname{FRCP}(C)^{2}$ \\ I Department of Family Medicine, Dalhousie University, Halifax, NS, Canada \\ 2 Department of Anesthesia, Pain Management \& Perioperative Medicine, Department of Critical Care, \\ Dalhousie University, Halifax, NS, Canada
}

\begin{abstract}
Takotsubo cardiomyopathy is a stress induced, transient cardiomyopathy that commonly presents with regional left ventricular akinesis or hypokinesis. The perioperative incidence of Takotsubo is estimated at 1:6700 cases and as such, should be familiar to practicing anesthesiologists. Point of care ultrasonography is a valuable tool for the evaluation of cardiac function and can be utilized in the perioperative setting by anesthesia staff. With some basic training in ultrasonography, one can quickly assess volume status and cardiac function, among other things. We present the case of a 73-year-old male undergoing an elective rotator cuff repair with an interscalene block under general anesthesia. Immediately following induction, the patient had a pulseless electrical activity arrest. Following immediate resuscitation, point of care ultrasonography was used to delineate the cause of the arrest and guide immediate management. Features consistent with Takotsubo cardiomyopathy - including regional left ventricular wall motion abnormalities with associated low ejection fraction - were identified on early ultrasound. The patient made a successful recovery and repeat cardiac imaging showed resolution of the wall motion abnormalities.
\end{abstract}

$\mathrm{P}$ oint of care ultrasound (POCUS) is an increasingly relevant imaging modality across a number of different medical specialties, including intensive care, emergency medicine, and anesthesia. The advent of smaller, handheld devices has allowed the use of this tool to expand across disciplines. There is an increasing body of literature showing that cardiac POCUS can be used to reliably identify and rule out a number of important pathologies, even when performed by a noncardiologist. ${ }^{1}$ While not routinely used in all centers currently, POCUS has been shown to be a useful tool to the anesthesiologist in assessment of a perioperative patient. Canty \& Royse demonstrated its utility in a small study of 97 patients where anesthesiologists performed 87 transthoracic and 14 transesophageal studies. $^{2}$ These assessments resulted in three cancelled surgeries, seven referrals to cardiology, and four patients having a change in their surgical plan. Additionally, there were 34 changes in the anesthetic management of patients as a result of this point of care study. While this tool is certainly of use in many areas of anesthesia, its full role in both practice and medical education has yet to be fully elucidated. ${ }^{3}$

Takotsubo cardiomyopathy is a transient cardiac condition whereby there is circumferential akinesis and ballooning of the apex of the left ventricle, usually triggered by a stressful event (physical or emotional), which can result in acute heart failure. The name comes from the Japanese word for an octopus trap, the outline of which closely resembles the left ventricle in systole. ${ }^{4,5}$ While the majority of cases involve females, younger males seem to be at higher risk in the perioperative period. ${ }^{6,7}$ The prevalence of Takotsubo cardiomyopathy among perioperative patients is estimated at 1:6700, and therefore should be understood by the practicing anesthesiologist. $^{\text {? }}$

We describe a case in which a patient presented to the operating room for an elective orthopedic procedure and developed a pulseless electrical activity arrest. Early use of POCUS by the anesthesiologist demonstrated findings keeping with Takotsubo cardiomyopathy and was useful in guiding early management.

\section{Case Summary}

The patient described was a 73-year-old male undergoing an elective repair of his left rotator cuff. Past medical history was remarkable for atrial fibrillation, obstructive sleep apnea, and hypothyroidism. His medications included dabigatran $150 \mathrm{mg}$ twice daily, levothyroxine $0.05 \mathrm{mg}$ once daily and sotalol $40 \mathrm{mg}$ once daily. He had a reported allergy to sulfamethoxazole.

After discussion with the patient, an interscalene block was performed using $1 \mathrm{mg}$ of midazolam for sedation and $20 \mathrm{~mL}$ of ropivicaine $0.5 \%$ for anesthesia. Prior to exiting the block room, the patient's blood pressure was $100 / 50 \mathrm{mmHg}$ and heart rate was 60 beats per minute. The patient arrived in the operating room approximately 20 minutes following the administration of the interscalene block. Induction took place five minutes later using $100 \mu \mathrm{g}$ of fentanyl, $1 \mathrm{mg}$ of midazolam, $130 \mathrm{mg}$ of propofol, $100 \mu \mathrm{g}$ of remifentanyl and $40 \mathrm{mg}$ of rocuronium.

Immediately following induction, the patients' blood pressure was noted to be $34 / 25 \mathrm{mmHg}$ and 
the patient had a pulseless electrical activity arrest. Cardiopulmonary resuscitation was initiated immediately and continued for three minutes before return of spontaneous circulation. During the resuscitation, the patient received $1 \mathrm{mg}$ of epinephrine, $300 \mu \mathrm{g}$ of phenylephrine and $0.8 \mathrm{mg}$ of vasopressin. An electrocardiogram obtained after the event demonstrated slow atrial fibrillation.

In the post-anesthesia care unit nursing staff were unable to obtain a blood pressure measurement and an anesthesia staff with special interest in POCUS performed a bedside transthoracic echocardiogram to help delineate the cause of the patient's arrest. A brief initial assessment of the inferior vena cava identified venous stasis and poor circulatory effort, and directed early initiation of peripheral inotropes. Once resuscitated, a more complete exam was performed which demonstrated widespread left ventricular akinesis (Figure 1), and an estimated ejection fraction of less than $20 \%$. The right ventricle function was felt to be within normal parameters.

High sensitivity troponin following the arrest was measured at $48 \mathrm{ng} / \mathrm{L}$, and rose to $422 \mathrm{ng} / \mathrm{L}$ the following day. N-terminal pro-brain natriuretic peptide (NT-proBNP) was elevated at $5714 \mathrm{pg} / \mathrm{mL}$ one day following the event. Coronary angiography demonstrated focal $70 \%$ stenosis of the distal circumflex artery with 40-50\% narrowing of the first medium obtuse branch. The left main coronary artery had a minor attenuation to $20 \%$ and the left anterior descending artery showed minor attenuation to $30-40 \%$. The cardiology service felt that this degree of coronary artery disease could not explain the severe degree of cardiac dysfunction that was observed.

A formal echocardiogram done three days later demonstrated a hyperdynamic left ventricle with an estimated ejection fraction of $65 \%$ and high filling pressures. The right ventricle was normal in size and systolic function. Repeat imaging at eight months was largely unchanged.

\section{Discussion}

While the diagnostic criteria for Takotsubo cardiomyopathy are not widely agreed upon, we utilized the diagnostic criteria described by The Mayo Clinic for diagnosis. ${ }^{8}$ In this case, the stressful trigger was the patient's cardiac arrest and subsequent resuscitation. A recent systematic review identified 41 cases of Takotsubo cardiomyopathy related to epinephrine use. ${ }^{9}$ While we are unable to identify causation, our case is another example of a potential association between administration of epinephrine and Takotsubo.

POCUS performed by anesthesia staff demonstrated left ventricular akinesis with sparing of the base of the heart. This widespread involvement of the left ventricle extended past the territory of a single coronary artery and angiography identified neither complete obstruction nor plaque rupture. This pattern of ventricular wall involvement is the classically described presentation of Takotsubo cardiomyopathy. We also observed changes in cardiac troponin in keeping with the diagnostic criteria. The presentation was not in keeping with myocarditis or pheochromocytoma nor did investigations suggest either diagnosis.

Fortunately, the patient made a complete recovery

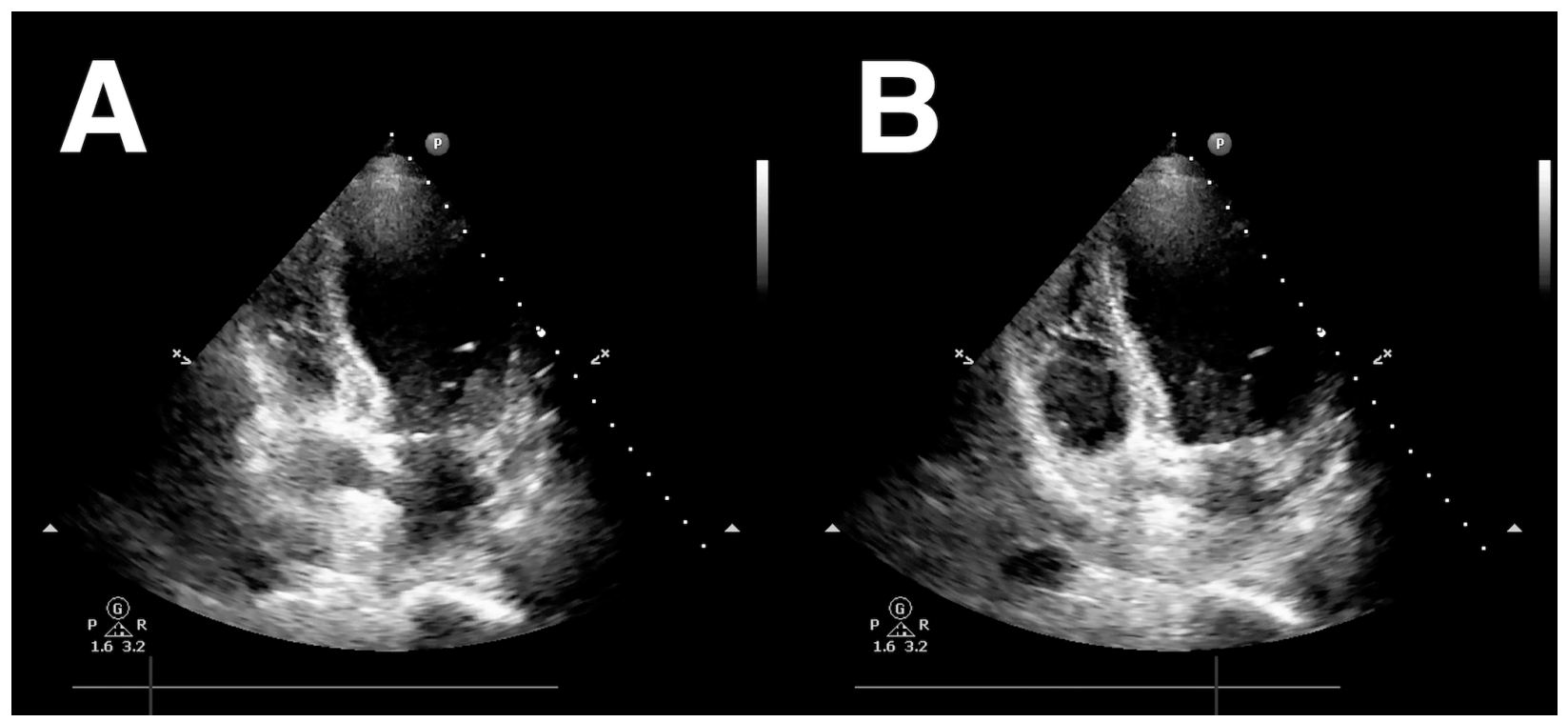

Figure 1. Point of care ultrasonography images captured in the post anesthesia care unit following resuscitation. Note is made of the left ventricular akinesis with ballooning of the mid and distal left ventricle in systole that is typical of Takotsubo cardiomyopathy. (A) Apical four chamber view during systole. (B) Apical four chamber view during diastole. 
and follow-up cardiac imaging demonstrated resolution of left ventricular function. In our case, early access to bedside echocardiography was the first suggestion that this was a case of Takotsubo cardiomyopathy and was helpful in guiding initial treatment. Early use of ultrasound quickly alerted the staff to the severity of the left ventricular dysfunction and prompted the decision to proceed immediately with cardiac catheterization.

As this condition is most likely under-diagnosed it is important to have a tool that can aid in early identification. ${ }^{4,7}$ Access to and training in POCUS is a useful tool in the hands of the anesthesiologist, and is applicable to other fields of medicine.

\section{Conclusion}

Takotsubo cardiomyopathy is a potentially serious cardiac condition, and likely occurs more commonly in the perioperative period than recognized. ${ }^{7}$ We believe that POCUS is an excellent tool for early evaluation of cardiac dysfunction that may help to guide management of hemodynamic instability. The literature suggests that bedside evaluation of cardiac function is a skill that is easily taught within a reasonable time frame and we feel it can change patient management. ${ }^{1,3}$ We believe the application of POCUS as a point of care test can be an irreplaceable tool in the practice of modern anesthesiology.

Patient consent was obtained for the preparation and submission of this report for publication.

\section{References}

1. Haji DL, Royse A, Royse CF. Review article: Clinical impact of non-cardiologist-performed transthoracic echocardiography in emergency medicine, intensive care medicine and anaesthesia. Emerg Med Australas 2013;25(1):4-12.

2. Canty DJ, Royse CF. Audit of anaesthetist-performed echocardiography on perioperative management decisions for non-cardiac surgery. $\mathrm{Br} \mathrm{J}$ Anaesth 2009;103(3):352-8.

3. Deshpande R, Montealegre-Gallegos M, Matyal R, Belani K, Chawla N. Training the anesthesiologist in point-of-care ultrasound. Int Anesthesiol Clin 2016;54(1):71-93.

4. Lyon A, Bossone E, Schneider B, Sechtem U, Citro R, Underwood, et al. Current state of knowledge on Takotsubo syndrome: a position statement from the Taskforce on Takotsubo Syndrome of the Heart Failure Association of the European Society of Cardiology. Eur J Heart Fail 2016;18(1):8-27.

5. Ono R, Falcão LM. Takotsubo cardiomyopathy systematic review: Pathophysiologic process, clinical presentation and diagnostic approach to Takotsubo cardiomyopathy. Int J Cardiol 2016;209:196-205.

6. Templin C, Ghadri JR, Diekmann J, Napp LC, Bataiosu DR, Jaguszewski $\mathrm{M}$, et al. Clinical features and outcomes of Takotsubo (Stress) cardiomyopathy. N Engl J Med 2015;373(10):929-38.

7. Hessel EA. Takotsubo cardiomyopathy and its relevance to anesthesiology: a narrative review. Can J Anesth 2016;63(9):1059-74.

8. Madhavan M, Prasad A. Proposed Mayo Clinic criteria for the diagnosis of Tako-Tsubo cardiomyopathy and long-term prognosis. Herz 2010;35:240-44.

9. Nazir S, Lohani S, Tachamo N, Ghimire S, Poudel DR, Donato A. Takotsubo cardiomyopathy associated with epinephrine use: A systematic review and meta-analysis. Int. J. Cardiol 2017;229:67-70.

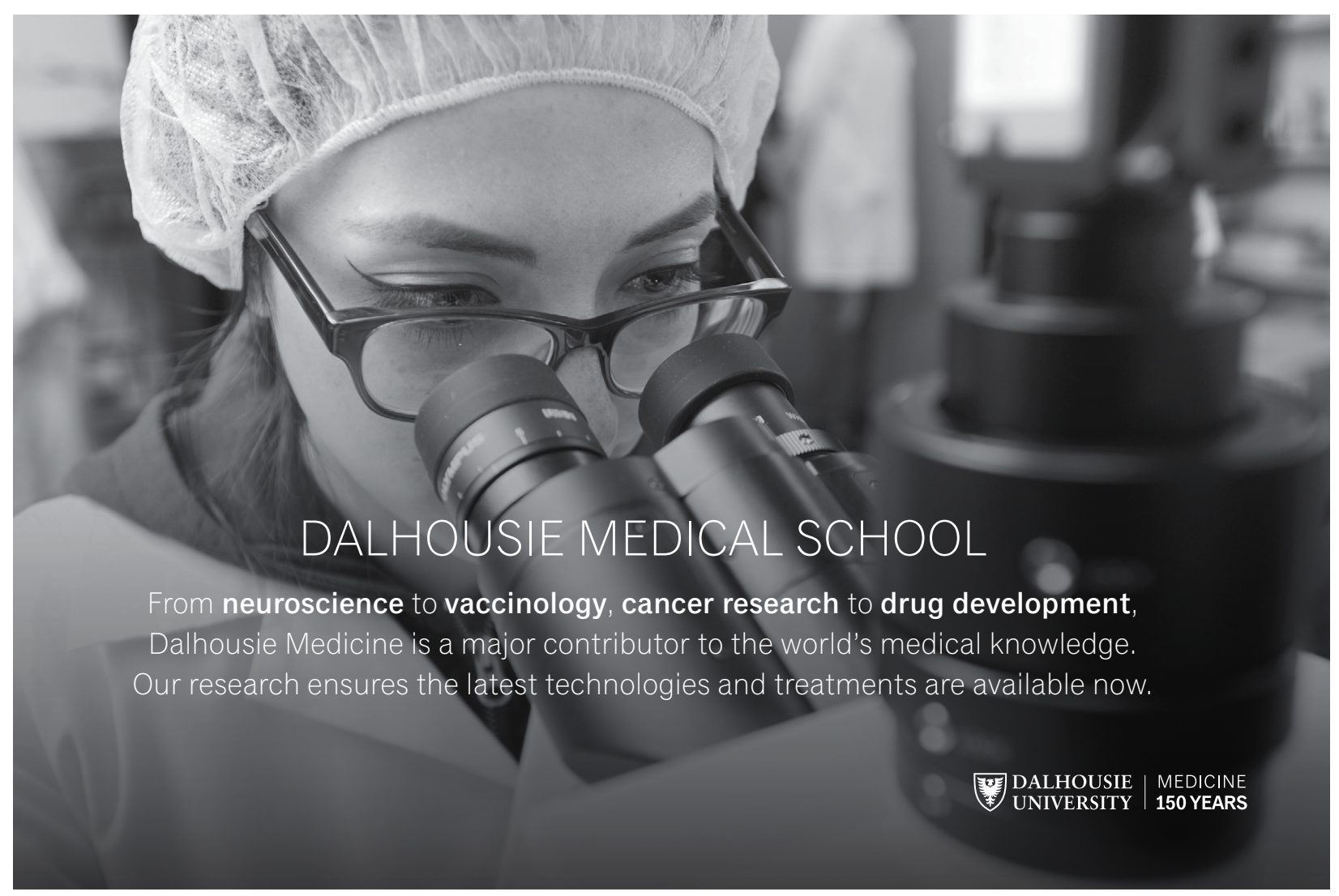

